results in two alternate alleles (19). The insertion of an additional guanine increases transcriptional activity due to the creation of a core binding site for a transcription factor from the Ets family (19). It would be interesting to test whether this polymorphism favors tissue destruction and enhances risk of disease progression in TB. Supporting this idea, a recent study reported that the presence of the $-1607 \mathrm{G}$ allele was significantly higher in TB patients with advanced pulmonary fibrosis compared with that in those with minimal fibrosis (20).

\section{Conclusions}

Taken together, an emerging model (Figure 1) for the role of MMPs in TB is that M. tuberculosis induces tissue remodeling via induction of MMP-9 to establish itself in the host. Once M. tuberculosis is established within the granuloma, maintaining intact granulomas is beneficial to the host, since it keeps the pathogen under check. During reactivation of a latent infection, excessive MMP-1 secretion leads to matrix degradation and cavitation. Much remains to be learned regarding the spatial and temporal regulation of specific MMPs during lung remodeling in TB. Nonetheless, the work of Freidland's group, along with other recent publications, suggest that MMPs represent viable therapeutic targets to control M. tuberculosis replication soon after infection by preventing nascent granulomas from maturing and by blocking matrix degradation and cavitary disease.

\section{Acknowledgments}

The author's research program is supported by NIH grants AI084822, AI069395, and AI065663.

Address correspondence to: Padmini Salgame, UMDNJ-New Jersey Medical School, Center for Emerging Pathogens, South Orange Avenue, MSB A902, Newark, New Jersey 07101, USA. Phone: 973.972.8647; Fax: 973.972.0713; E-mail: salgampa@ umdnj.edu.

1. Saunders BM, Britton WJ. Life and death in the granuloma: immunopathology of tuberculosis. Immunol Cell Biol. 2007;85(2):103-111.

2. Scott-Browne JP, et al. Expansion and function of Foxp3-expressing $\mathrm{T}$ regulatory cells during tuberculosis. J Exp Med. 2007;204(9):2159-2169.

3. Whalen CC, et al. A trial of three regimens to prevent tuberculosis in Ugandan adults infected with the human immunodeficiency virus. Uganda-Case Western Reserve University Research Collaboration. N Engl J Med. 1997;337(12):801-808.

4. Gardam MA, et al. Anti-tumour necrosis factor agents and tuberculosis risk: mechanisms of action and clinical management. Lancet Infect Dis. 2003;3(3):148-155.

5. Gross J, Lapiere CM. Collagenolytic activity in amphibian tissues: a tissue culture assay. Proc Natl Acad Sci U S A. 1962;48:1014-1022.

6. Parks WC, Shapiro SD. Matrix metalloproteinases in lung biology. Respir Res. 2001;2(1):10-19.

7. Parks WC, Wilson CL, Lopez-Boado YS. Matrix metalloproteinases as modulators of inflammation and innate immunity. Nat Rev Immunol. 2004;4(8):617-629

8. Rivera-Marrero CA, Schuyler W, Roser S, Roman J. Induction of MMP-9 mediated gelatinolytic activity in human monocytic cells by cell wall components of Mycobacterium tuberculosis. Microb Patbog. 2000;29(4):231-244.

9. Quiding-Jarbrink M, Smith DA, Bancroft GJ. Production of matrix metalloproteinases in response to mycobacterial infection. Infect Immun. 2001;69(9):5661-5670.
10. Chang JC, Wysocki A, Tchou-Wong KM, Moskowitz N, Zhang Y, Rom WN. Effect of Mycobacterium tuberculosis and its components on macrophages and the release of matrix metalloproteinases. Tho$\operatorname{rax} .1996 ; 51(3): 306-311$

11. Hernandez-Pando R, Orozco H, Arriaga K, Pavon L, Rook G. Treatment with BB-94, a broad spectrum inhibitor of zinc-dependent metalloproteinases, causes deviation of the cytokine profile towards type-2 in experimental pulmonary tuberculosis in Balb/c mice. Int J Exp Pathol. 2000;81(3):199-209.

12. Izzo AA, Izzo LS, Kasimos J, Majka S. A matrix metalloproteinase inhibitor promotes granuloma formation during the early phase of Mycobacterium tuberculosis pulmonary infection. Tuberculosis. 2004;84(6):387-396.

13. Taylor JL, et al. Role for matrix metalloproteinase 9 in granuloma formation during pulmonary Mycobacterium tuberculosis infection. Infect Immun. 2006;74(11):6135-6144.

14. Volkman HE, Pozos TC, Zheng J, Davis JM, Rawls JF, Ramakrishnan L. Tuberculous granuloma induction via interaction of a bacterial secreted protein with host epithelium. Science. 2010;327(5964):466-469.

15. Hrabec E, Strek M, Zieba M, Kwiatkowska S, Hrabec Z. Circulation level of matrix metalloproteinase-9 is correlated with disease severity in tuberculosis patients. Int J Tuberc Lung Dis. 2002;6(8):713-719.

16. Price NM, Gilman RH, Uddin J, Recavarren S, Friedland JS. Unopposed matrix metalloproteinase- 9 expression in human tuberculous granuloma and the role of TNF-alpha-dependent monocyte networks. J Immunol. 2003;171(10):5579-5586.

17. Elkington P, et al. MMP-1 drives immunopathology in human tuberculosis and transgenic mice. J Clin Invest. 2011;121(5):1827-1833.

18. Pichugin AV, Yan BS, Sloutsky A, Kobzik L, Kramnik I. Dominant role of the sst 1 locus in pathogenesis of necrotizing lung granulomas during chronic tuberculosis infection and reactivation in genetically resistant hosts. Am J Pathol. 2009;174(6):2190-2201.

19. Rutter JL, et al. A single nucleotide polymorphism in the matrix metalloproteinase- 1 promoter creates an Ets binding site and augments transcription. Cancer Res. 1998;58(23):5321-5325.

20. Wang $\mathrm{CH}$, et al. MMP-1(-1607G) polymorphism as a risk factor for fibrosis after pulmonary tuberculosis in Taiwan. Int J Tuberc Lung Dis. 2010;14(5):627-634.

\title{
Fibulin-5: two for the price of one maintaining pelvic support
}

\author{
Gina M. Northington
}

Department of Obstetrics and Gynecology, University of Pennsylvania School of Medicine, Philadelphia, Pennsylvania, USA.

\begin{abstract}
Pelvic organ prolapse (POP) is a disabling disorder in women characterized by a loss of pelvic floor support leading to the herniation of the uterus into or through the vagina. POP is a complex problem that likely involves multiple mechanisms, and available therapies are limited. In this issue of the JCI, Budatha et al. explore the dual role carried out by fibulin-5 in facilitating the assembly of normal elastic fibers and inhibiting MMP-9 activity, revealing a new mechanism critical to the maintenance of pelvic organ support.
\end{abstract}

Conflict of interest: The author has declared that no conflict of interest exists.

Citation for this article: J Clin Invest. doi:10.1172/ JCI57438.
Pelvic organ prolapse (POP) is a major cause of morbidity among women in the United States. It occurs in approximately $35 \%$ of the adult female population, and the incidence increases with age (1). The impact of POP on women is significant, including sexual dysfunction, social isolation, depression, and poor body image (2). The etiology is multifactorial, with a history of vaginal childbirth being the most notable risk factor $(3,4)$. It is estimated that $11 \%-19 \%$ of women will require surgical repair in their lifetime to treat prolapse, and approximately one-third of these will require additional surgery for recurrent disease (5); thus it is important to define the molecular and cellular mecha- 


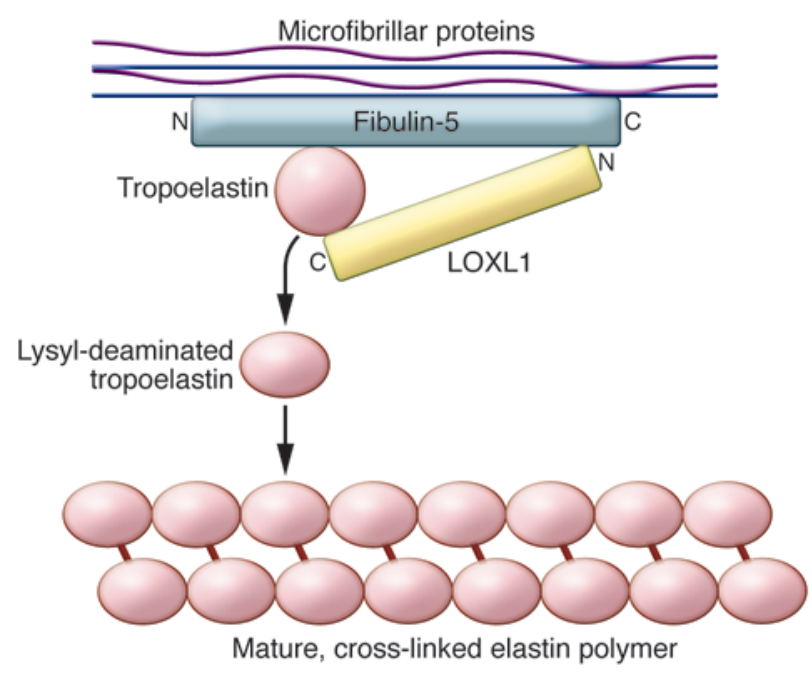

nisms of POP. Because of the difficulty of procuring human tissue and the expense associated with studying POP in primates (6), rodent models of elastinopathies have been used to study this disease (7-9). While rodents are quadrupeds and, by definition, have different forces operating on the pelvic floor, the connective tissue anatomy is grossly similar to that seen in humans (10). Although pelvic floor injury is recognized as a common antecedent to the development of POP, an improved understanding of the subsequent cellular and genetic alterations in connective tissue is required to improve therapeutic strategies. In this issue of the JCI, Budatha et al. (11) exploit murine genetic models to develop a unifying molecular mechanism of abnormal elastic fiber homeostasis leading to POP and identify fibulin-5 as the central player in the pathophysiology of this disorder (12).

\section{Elastinopathy in pelvic organ prolapse}

Because women suffering from connective tissue disorders such as Marfan or Ehlers-Danlos syndromes have high rates of spontaneous pelvic organ prolapse (13), considerable interest has been directed at understanding alterations in connective tissue homeostasis that contribute to the etiology of prolapse. The pelvic floor is supported by an ECM composed of collagen, elastic fibers, and proteoglycans, and several published reports associate POP with changes in these ECM components and in the activity of MMPs (14-16).

MMPs play an important role in tissue remodeling and collectively form a 26member family of proteases that are mainly responsible for proteolytic degradation of the ECM proteins, including collagens, gelatins, and elastin. Although degradation has been shown to be an important part of MMP function, recent data suggest that MMPs can also affect cellular growth and behavior through the interaction between MMP substrates with ECMindependent factors, including growth factors, cytokines, adhesion receptors, and cell surface proteoglycans (17). Previous studies have identified MMP-2 and -9 as the primary elastolytic enzymes involved in the pathogenesis of POP (3). Utilizing zymography, Jackson et al. demonstrated increased active MMP-2 and -9 in women with POP compared with controls (14). Other groups have demonstrated a similar increase in the active form of MMP-9 in association with POP (3). Interestingly, an increase in MMP-9 activity does not necessarily correspond to an increased transcript level in prolapsed vaginal tissue, suggesting that posttranscriptional regulation of MMP-9 occurs in POP (15). Taken together, these data signify that increased ECM turnover is an essential process in this disorder.

While mature, insoluble elastin is generally stable after development throughout the life span (18), elastic fibers in the female reproductive tract undergo frequent turnover under normal physiologic conditions. Elastin is an insoluble and durable cross-linked array synthesized by joining soluble tropoelastin protein molecules in a reaction catalyzed by lysyl oxidase (LOX) (Figure 1) and provides expansion and recoil in response to tension, which supports the pelvic floor during parturition. Elastic fibers normally degrade during labor and are resynthesized following

\section{Figure 1}

Model of elastogenesis. $\mathrm{N}$-terminal region of LOXL1 binds to C-terminal domain of fibulin- 5 to activate tropoelastin by converting to lysyldeaminated form. Covalent cross-linking of tropoelastin occurs to form mature elastin. There are additional regulatory processes in elastogenesis that are tissue specific. vaginal delivery (19); this process is under hormonal control and is mediated by age (20). Wieslander et al. (19) demonstrated increased MMP-2 and MMP-9 enzyme activities with a corresponding decrease in protease inhibitors (TIMP2, cystatin C, and $\alpha-1$ antitrypsin) in genetically normal mice 48 hours after delivery of pups. It is theorized that abnormalities in this normal process of degradation and renewal could lead to POP as women age.

\section{Murine models of POP}

LOXs are a family of five enzymes that catalyze the cross-linking of collagen and elastin (21). LOX-like 1 protein (LOXL1) appears to be the most important factor in vaginal tissue remodeling that occurs after parturition and in POP associated with aging (20). LOXL1-knockout mice (Loxl1-/-) demonstrate multiple elastinopathies early in life but do not develop spontaneous POP until after 12 weeks of age or delivery of first and second litters (7). Loxl1 ${ }^{-/-}$mice have significantly lower vaginal elastin content and, together with absent normal fluctuations in LOXL1 throughout pregnancy and parturition, this leads to abnormal renewal of the ECM following delivery compared with WT animals (7). These studies suggest that synthesis and degradation of elastic fibers both play a role in vaginal wall remodeling.

Another family of proteins important in elastic fiber matrix assembly and function are fibulins. Fibulins are secreted glycoproteins that become incorporated into a fibrillar extracellular matrix. The seven known members of the fibulin family (fibulins 1-7) have a series of EGF-like domains followed by a C-terminal "fibulin-type" domain (22). Both fibulin-3 and 
A Fbln5 (WT)

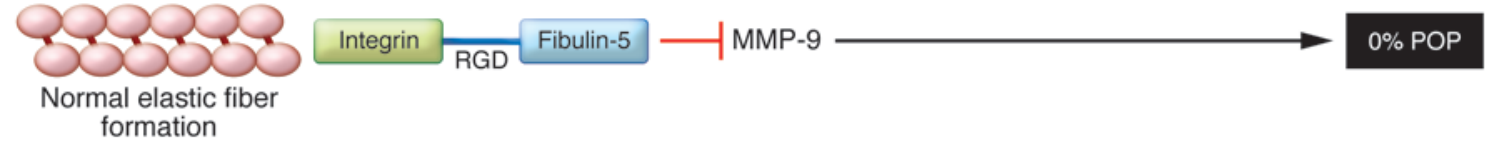

B Fbln5 ${ }^{\text {GGE/RGE }}$

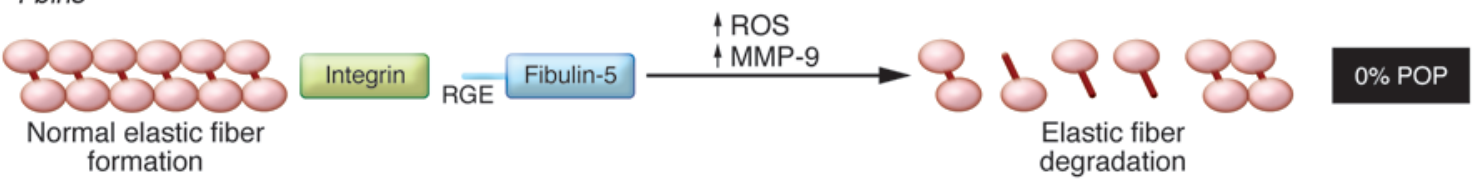

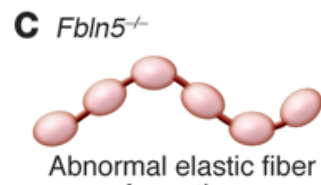
formation

D Fbln5 $5^{--} \mathrm{MMPO}^{--}$(DKO)

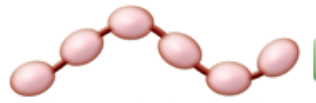

Abnormal elastic fiber formation
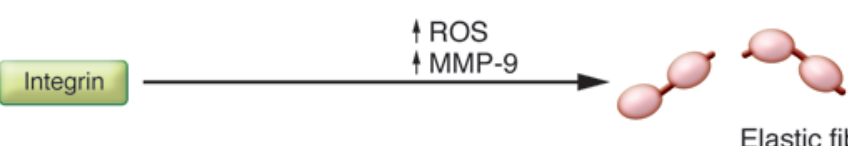

Elastic fiber degradation

Integrin
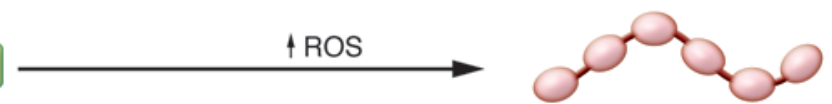

$40 \%$ POP

\section{Figure 2}

Role of Fibulin 5 in POP. (A) In Fbln5 $5^{+/+}$(WT) mice, there is normal elastic fiber synthesis. Fbln5 interacts with integrin, inhibiting MMP-9. (B) In FbIn5 RGE/RGE animals, there is normal elastic fiber formation and increased MMP-9 activity. Similar to the WT, POP does not develop in these animals. (C) Fbln5 $5^{-/-}$animals develop abnormal elastic fibers, increased ROS, and increased MMP-9 activity. POP develops in $96 \%$ of $F b / n 5^{-/-}$ animals. (D) Fbln5 $5^{--} M M P-9^{-/-}$(DKO) animals have abnormal elastic fibers. Only $40 \%$ of animals developed POP, suggesting the importance of both abnormal elastic fiber synthesis and increased MMP-9 activity in development of this disease.

fibulin-5 have been implicated as having a role in maintaining pelvic support $(8,9)$. Fibulin-3-knockout mice (Fbln $3^{-1-}$ ) have abdominal wall fascial defects and variably develop POP that increases with age. Moreover, the Fbln $3^{-/-}$animals that exhibit POP also have increased levels of active MMP-2 and MMP-9 compared with Fbln3 $3^{-/-}$animals without prolapse or WT animals (9). However, these animals experience normal tissue recovery following parturition. This latter finding could be related to the observed compensatory increase in mRNA transcripts for protease inhibitors (TIMP1, TIMP3), fibulin-5, and tropoelastin (9).

Fibulin-5-knockout mice (Fbln $5^{-/-}$) consistently develop prolapse with advancing age: $91 \%$ of $F b \ln 5^{-1-}$ animals develop POP by 6 months of age, while this is true of only $50 \%$ and $27 \%$ of older Loxl1 $1^{-/}$and Fbln $3^{-/-}$animals, respectively (7-9). Additionally, increased monocytic infiltration in the vaginal wall during labor seen in $\mathrm{Fbln5} 5^{-/}$animals may indicate increased elastolytic activity, further suggesting that both abnormal synthesis and increased degradation are necessary to develop POP (8). Given the aforementioned findings in both Fbln5 $5^{-/-}$and Loxl1 $1^{-/-}$animals, Budatha et al. sought to further explain the interactions between fibulin-5 and LOXL1 in the pathogenesis of POP (11).

Fibulin-5 promotes cell adhesion by interacting with integrins via its RGD motif. Budatha et al. generated mice homozygous for alleles of Fbln 5 in which the RGD motif was mutated (Fbln5RGE) and found that these mice had elastic fibers and ECM protein content that was comparable to that of WT animals (11). In contrast to the Fbln $5^{-/-}$animals, POP was not observed in the Fbln $5^{R G E / R G E}$ animals after pregnancy or with advancing age, suggesting that integrin-binding activity of fibulin-5 does not affect normal elastic fiber formation (Figure 2). The authors further demonstrated a significant increase in MMP-9 activity in vaginal tissues from the $F b \ln 5^{R G E / R G E}$ and Fbln $5^{-/-}$animals compared with WT. They determined that fibulin 5 indirectly medi- ates the upregulation of MMP-9 in an RGD-dependent manner. ROS also upregulate MMP-9 in vaginal stromal cells from both fibulin mutant mouse models, suggesting that the fibulin-5 integrin interaction is a mediator in the pathway between ROS and MMP-9 activity in the vaginal wall (Figure 2).

What is most interesting about this study is that, although $F b \ln 5^{R G E / R G E}$ mice do not appear to develop POP or abnormal elastic fiber assembly, they do develop a milder form of disease when LOX activity is blocked by treating animals with $\beta$-aminopropionitrile (BAPN). If this is due to impaired de novo elastin synthesis, as the authors suggest, it would be interesting to see whether more severe POP develops in animals treated with BAPN for longer periods of time. To further demonstrate the necessity of both MMP-9 activity and abnormal elastic fiber synthesis for POP, Budatha et al. generated mice that were deficient in both MMP-9 and fibulin-5 to form a double KO (DKO) model (11). Only $42 \%$ of the DKO mice developed POP, 
while $95 \%$ of the $\mathrm{Fbln}^{-/-}$animals developed the disorder, strengthening the argument that MMP-9 upregulation accounts for a significant part of the pelvic floor dysfunction in $\mathrm{Fbln}^{-/-}$mice.

\section{Concluding remarks}

There is increasing evidence that POP results from alterations in the relative balance between synthesis and degradation of the ECM in pelvic tissues. In this paper, Budatha et al. have elegantly established a dual role for fibulin-5 in the regulation of elastic fiber homeostasis (11). They demonstrate that fibulin-5 not only facilitates the normal assembly of elastic fibers, but also inhibits MMP-9 through RGD-dependent integrin-binding. In addition, they provide evidence that both abnormal elastic fibers and upregulation of MMP-9 are necessary and sufficient for the full manifestation of POP in murine models.

Although these results have generated important information about the elastinopathic pathways involved in POP, several questions remain. It is still not known how hormonal and aging mechanisms have an impact on these pathways. This is highlighted by the human studies included in this paper. The researchers demonstrated that although MMP-9 was significantly increased in women with prolapse compared with controls, the difference among postmenopausal women (prolapse vs. control) was a large 23 -fold change while, among the premenopausal women, there was only a modest 3-fold change. These results, and those of other groups (3), suggest that hormonal modulation is an important factor and should be considered in all molecular pathways that lead to POP.

Other human studies have suggested that alterations in genes responsible for elastic fiber homeostasis are more common in women with clinical manifestations of POP (23), and family history is a known risk factor for the development of this dis- ease (24). It would be important to understand how genetic susceptibility plays into the elastinopathic pathways identified in murine models. Further research is needed to phenotype individuals to determine who is at risk for developing POP primarily or to identify those at risk of recurrence after standard therapies.

\section{Acknowledgments}

The author's work is supported by the Women's Reproductive Health Research Award NIH/National Institute of Child Health and Human Development (K12HD00165-11).

Address correspondence to: Gina M. Northington, Department of Obstetrics and Gynecology, Division of Urogynecology and Reconstructive Pelvic Surgery, The Hospital of the University of Pennsylvania, 3400 Spruce St., 1000 Ravdin Courtyard, Philadelphia, Pennsylvania 19104, USA. Phone: 215.662.6361; Fax: 215.662.7929; E-mail: gnorthington@obgyn.upenn.edu.

1. Lawrence JM, Lukacz ES, Nager CW, Hsu JW, Luber KM. Prevalence and co-occurrence of pelvic floor disorders in community-dwelling women. Obstet Gynecol. 2008;111(3):678-685.

2. Lowder JL, Ghetti C, Nikolajski C, Oliphant SS, Zyczynski HM. Body image perceptions in women with pelvic organ prolapse: a qualitative study [published online ahead of print February 1, 2011]. Am J Obstet Gynecol. doi:10.1016/j.ajog.2010.12.024.

3. Word RA, Pathi S, Schaffer JI. Pathophysiology of pelvic organ prolapse. Obstet Gynecol Clin North Am. 2009;36(3):521-539.

4. Lukacz ES, Lawrence JM, Contreras R, Nager CW, Luber KM. Parity, mode of delivery, and pelvic floor disorders. Obstet Gynecol. 2006;107(6):1253-1260.

5. Smith FJ, Holman CD, Moorin RE, Tsokos N. Lifetime risk of undergoing surgery for pelvic organ prolapse. Obstet Gynecol. 2010;116(5):1096-1100.

6. Mattson JA, Kuehl TJ, Yandell PM, Pierce LM, Coates KW. Evaluation of the aged female baboon as a model of pelvic organ prolapse and pelvic reconstructive surgery. Am J Obstet Gynecol. 2005;192(5):1395-1398.

7. Liu X, Zhao Y, Pawlyk B, Damaser M, Li T. Failure of elastic fiber homeostasis leads to pelvic floor disorders. Am J Pathol. 2006;168(2):519-528.

8. Drewes PG, et al. Pelvic organ prolapse in fibulin5 knockout mice: pregnancy-induced changes in elastic fiber homeostasis in mouse vagina. Am J Pathol. 2007;170(2):578-589.

9. Rahn DD, et al. Failure of pelvic organ support in mice deficient in fibulin-3. Am J Pathol. 2009;174(1):206-215.

10. Moalli PA, et al. A rat model to study the structural properties of the vagina and its supportive tissues. Am J Obstet Gynecol. 2005;192(1):80-88.

11. Budatha M, et al. Extracellular matrix proteases contribute to progression of pelvic organ prolapse in mice and humans. J Clin Invest. 2011; 121(5):2048-2059.

12. Yanagisawa H, Schluterman MK, Brekken RA. Fibulin-5, an integrin-binding matricellular protein: its function in development and disease. J Cell Commun Signal. 2009;3(3-4):337-347.

13. Carley ME, Schaffer J. Urinary incontinence and pelvic organ prolapse in women with Marfan or Ehlers Danlos syndrome. Am J Obstet Gynecol. 2000;182(5):1021-1023.

14. Jackson SR, Avery NC, Tarlton JF, Eckford SD, Abrams P, Bailey AJ. Changes in metabolism of collagen in genitourinary prolapse. Lancet. 1996;347(9016):1658-1661.

15. Chen BH, Wen Y, Li H, Polan ML. Collagen metabolism and turnover in women with stress urinary incontinence and pelvic prolapse. Int Urogynecol J Pelvic Floor Dysfunct. 2002;13(2):80-87.

16. Moalli PA, Shand SH, Zyczynski HM, Gordy SC, Meyn LA. Remodeling of vaginal connective tissue in patients with prolapse Obstet Gynecol. 2005; 106(5 pt 1):953-963.

17. Stamenkovic I. Extracellular matrix remodelling: the role of matrix metalloproteinases. $J$ Pathol. 2003;200(4):448-464.

18. Mecham RP, Davis EC. Elastic fiber structure and assembly. In: Yurchenko PD, Birk DE, Mecham RP, eds. Extracellular Matrix Assembly and Structure. San Diego, California, USA: Academic Press; 1994:281-314.

19. Wieslander CK, Marinis SI, Drewes PG, Keller PW, Acevedo JF, Word RA. Regulation of elastolytic proteases in the mouse vagina during pregnancy, parturition, and puerperium. Biol Reprod. 2008;78(3):521-528.

20. Liu X, et al. Elastic fiber homeostasis requires lysyl oxidase-like 1 protein. Nat Genet. 2004;36(2):178-182.

21. Kagan HM, Li W. Lysyl oxidase: properties, specificity, and biological roles inside and outside of the cell. J Cell Biochem. 2003;88(4):660-672.

22. Argraves WS, Greene LM, Cooley MA, Gallagher WM. Fibulins: physiological and disease perspectives. EMBO Rep. 2003;4(12):1127-1131.

23. Chen HY, Lin WY, Chen YH, Chen WC, Tsai FJ, Tsai CH. Matrix metalloproteinase-9 polymorphism and risk of pelvic organ prolapse in Taiwanese women. Eur J Obstet Gynecol Reprod Biol. 2010;149(2):222-224.

24. McLennan MT, Harris JK, Kariuki B, Meyer S. Family history as a risk factor for pelvic organ prolapse. Int Urogynecol J Pelvic Floor Dysfunct. 2008; 19(8):1063-1069. 